\title{
Spatial and Temporal Variation of Stable Isotopes in Precipitation across Costa Rica: An Analysis of Historic GNIP Records
}

\author{
Ricardo Sánchez-Murillo ${ }^{1,2^{*}}$, Germain Esquivel-Hernández ${ }^{1,3}$, Kristen Welsh $^{2,4}$, Erin S. Brooks ${ }^{5}$, \\ Jan Boll ${ }^{2,5}$, Rosa Alfaro-Solís ${ }^{1,3}$, Juan Valdés-González ${ }^{1,3}$ \\ ${ }^{1}$ Escuela de Química, Universidad Nacional, Heredia, Costa Rica; ${ }^{2}$ Waters of the West-Water Resources Program, University of \\ Idaho, Moscow, USA; ${ }^{3}$ Laboratorio de Química de la Atmósfera, Escuela de Química, Universidad Nacional, Heredia, Costa Rica; \\ ${ }^{4}$ Division of Research and Development, Centro Agronómico Tropical de Investigación y Enseñanza (CATIE), Turrialba, Costa Rica; \\ ${ }^{5}$ Department of Biological and Agricultural Engineering, University of Idaho, Moscow, USA. \\ Email: *sanc7767@vandals.uidaho.edu
}

Received September $1^{\text {st }}, 2013$; revised October $1^{\text {st }}, 2013$; accepted October $8^{\text {th }}, 2013$

Copyright (C) 2013 Ricardo Sánchez-Murillo et al. This is an open access article distributed under the Creative Commons Attribution License, which permits unrestricted use, distribution, and reproduction in any medium, provided the original work is properly cited.

\begin{abstract}
The location of Costa Rica on the Central American Isthmus creates unique microclimate systems that receive moisture inputs directly from the Caribbean Sea and the Pacific Ocean. In Costa Rica, stable isotope monitoring was conducted by the International Atomic Energy Agency and the World Meteorological Association as part of the worldwide effort entitled Global Network of Isotopes in Precipitation. Sampling campaigns were mainly comprised of monthly-integrated samples during intermittent years from 1990 to 2005. The main goal of this study was to determine spatial and temporal isotopic variations of meteoric waters in Costa Rica using historic records. Samples were grouped in four main regions: Nicoya Peninsula $\left(\delta^{2} \mathrm{H}=6.65 \delta^{18} \mathrm{O}-0.13 ; r^{2}=0.86\right)$; Pacific Coast $\left(\delta^{2} \mathrm{H}=7.60 \delta^{18} \mathrm{O}+7.95 ; r^{2}=0.99\right)$; Caribbean Slope $\left(\delta^{2} \mathrm{H}=6.97 \delta^{18} \mathrm{O}+4.97 ; r^{2}=0.97\right)$; and Central Valley $\left(\delta^{2} \mathrm{H}=7.94 \delta^{18} \mathrm{O}+10.38 ; r^{2}=0.98\right)$. The water meteoric line for Costa Rica can be defined as $\delta^{2} \mathrm{H}=7.61 \delta^{18} \mathrm{O}+7.40\left(r^{2}=0.98\right)$. The regression of precipitation amount and annual arithmetic means yields a slope of $-1.6 \% \delta^{18} \mathrm{O}$ per $100 \mathrm{~mm}$ of rain $\left(r^{2}=0.57\right)$ which corresponds with a temperature effect of $-0.37 \% \delta^{18} \mathrm{O} /{ }^{\circ} \mathrm{C}$. A strong correlation $\left(r^{2}=0.77\right)$ of $-2.0 \%$ o $\delta^{18} \mathrm{O}$ per km of elevation was found. Samples within the Nicoya Peninsula and Caribbean lowlands appear to be dominated by evaporation enrichment as shown in $d$-excess interpolation, especially during the dry months, likely resulting from small precipitation amounts. In the inter-mountainous region of the Central Valley and Pacific slope, complex moisture recycling processes may dominate isotopic variations. Generally, isotopic values tend to be more depleted as the rainy season progresses over the year. Air parcel back trajectories indicate that enriched isotopic compositions both in Turrialba and Monteverde are related to central Caribbean parental moisture and low rainfall intensities. Depleted events appear to be related to high rainfall amounts despite the parental origin of the moisture.
\end{abstract}

Keywords: Stable Isotopes; Costa Rica; Precipitation; GNIP; HYSPLIT Model

\section{Introduction}

Tropical regions cover approximately 50\% of Earth's landmass and are home to three-quarters of the human population. During the last decade, the scientific community, environmental institutions, governments, and communities have increased their awareness of tropical climate variability based on the premise that changes in regional and global circulation trends may lead to inten-

"Corresponding author. sification (i.e., greater rainfall intensity) or weakening (i.e., prolonged drought) of the hydrological cycle in particular regions [1]. For example, several studies have shown the potential high vulnerability of tropical ecosystems to increasing mean surface temperatures [2-4]. In addition, extreme climate events could be even more severe than those experienced to date [5]. Climate anomalies will increase the vulnerability of certain regions and communities to changes in magnitude, timing, and duration of hydrological responses. 
Understanding precipitation patterns, particularly how they differ spatially and temporally, will provide further information on regional and global hydrologic processes and enable better planning and preparation for future potential changes in climate. A better understanding of the factors that control precipitation patterns will inform stakeholders and environmental agencies so that they can prioritize efforts and resources in ungauged basins where potential future droughts or floods may drastically affect ecological assemblages and socio-economic activities. In Central America, many socio-economic activities, especially agriculture, tourism, and hydropower generation, are dependent on seasonal cycles of precipitation. Therefore, in order to determine the interannual variability in precipitation, it is necessary to describe the regional annual cycle precisely and examine the mechanisms that control it [6].

Across the globe, spatial variation of stable isotopes in the water cycle occurs due to the movement of air masses and fractionation during phase changes, such as condensation and evaporation, creating a directional spatial pattern across regions and continents [7]. Light stable isotope compositions of tropical meteoric waters have proven to be an important indicator of current climate variability [8-16]. In particular, $\delta^{18} \mathrm{O}$ values have provided novel insights on El Niño/Southern Oscillation dynamics [12,17-19]. In temperate regions, isotopic variations in precipitation frequently have been correlated with mean surface air temperatures [20-25], whereas, in the tropics, several authors have reported "the amount effect" as the main controlling factor [16,24,26,27]. These consistent processes create relatively strong spatial patterns of isotopic compositions across landscapes [28]. Large-scale regional and global patterns can be depicted through isoscapes that illustrate the spatial and temporal variation of isotopes over a geographic region.

Despite novel advances to understand stable isotope precipitation dynamics in the tropics, there is consensus among scientists in the published literature $[13,16,27]$ on the urgent need for long-term sampling networks that may lead to a better spatial and temporal resolution, particularly in mountainous regions such as the Central American Continental Divide (CACD). In the CACD orographic effects, moisture recycling and canopy interception, intense evapotranspiration, and microclimates are relevant factors in isotopic variation. Furthermore, recent studies have demonstrated that variations in the stable isotope composition of precipitation occur even between storm events [29-32], emphasizing the importance of event-based sampling.

Stable isotope research in Costa Rica has been limited to date with no studies conducted on a regional scale examining trends in precipitation. Lachniet and Patterson
(2002) [27] provided a preliminary evaluation of $\delta^{18} \mathrm{O}$ and $\delta^{2} \mathrm{H}$ in Costa Rican surface waters $(n=63$ from rivers and lakes). Their study found regional trends in the isotopic composition of surface waters inversely correlated to altitude ranges $\left(-1.4 \delta^{18} \mathrm{O} \% / \mathrm{km}\right)$ and evaporation enrichment, particularly in the northwestern region. Rhodes et al. (2006) studied the seasonal variation of isotopic composition in tropical montane forests of Monteverde's National Park located on the continental divide. Based on seasonal $d$-excess variations, the authors concluded that water evaporated from land is an important component of the water budget to the region during the transitional and dry seasons. Reynolds and Fraile (2009) [33] conducted a groundwater recharge study on one of the main aquifers of the Central Valley of Costa Rica that provides water to approximately $30 \%$ of the population. Based upon precipitation, well, and spring records they were able to identify local recharge areas mainly dominated by Pacific-originated storms. Greater understanding of spatial and temporal isotopic variations in tropical regions with similar topographic, vegetation, and climatic attributes can enhance hydrological studies of this nature.

Costa Rica's climate is mainly controlled by four phenomena: northeast trades-winds (i.e., alisios), the shifts of the Intertropical Convergence Zone (ITCZ), cold continental outbreaks, and indirect influence of $\mathrm{Ca}$ ribbean cyclones [34]. These circulation processes produce three distinct seasons. The wet season (May-October) corresponds to the time when the ITCZ travels over Costa Rica, and precipitation is characterized by heavy convective rain storms. The transitional (NovemberJanuary) and dry (February-April) seasons comprise the months when the ITCZ is located to the south of Costa Rica [35]. During the wet season, the air masses arriving in Costa Rica can be classified as continental winds, reaching Costa Rica's Central Valley from the Pacific Ocean. Most of these air masses move over industrial and urban areas of the country. In the dry season, trade winds bring air masses to Costa Rica from the Caribbean Sea, and they move over non-industrial and less populated areas. Air masses arriving over Costa Rica during the transitional season can be transported by continental or trade-winds, and a weakening in the trade winds reaching Costa Rica is usually observed during the afternoon. Due to the transport pattern of the air masses observed in Costa Rica throughout the year, the isotopic composition of precipitation in Costa Rica is expected to vary depending on its origin from either the Caribbean Sea or the Pacific Ocean and also on the air trajectory across the country (i.e. industrial-urban areas versus remote-forested areas). Annual precipitation varies from $\sim 1500 \mathrm{~mm}$ in the northwestern region and Nicoya Pen- 
insula to $\sim 7000 \mathrm{~mm}$ on the Caribbean side of the Talamanca range. Temperature seasonality is low. The mean annual temperatures vary from around $27^{\circ} \mathrm{C}$ on the coastal lowlands, $20^{\circ} \mathrm{C}$ in the Central Valley, and below $10^{\circ} \mathrm{C}$ at the summits of the highest mountain range ( $\sim 4000$ m.a.s.l.).

The Global Network for Isotope in Precipitation (GNIP) [36] data set provides isotopic values of precipitation from 46 stations across Costa Rica between 1990 and 2005 (intermittent collection over this period). Most of the reported stations were located within the Central Valley (greatest populated area) and the North Pacific region. Therefore, a considerable gap exists along the Pacific and Caribbean shores, continental divide, and the northern and southern regions. Herein, we present an analysis of the spatial and temporal variation of stable isotopes in meteoric waters in Costa Rica and determine which broad climatic or topographic factors may influence isotopic variations across the region. Results of this analysis provide baseline historical spatial-temporal characterization of meteoric waters in Costa Rica that has been lacking to date.

\section{Methods and Materials}

\subsection{Study Area}

The Central Valley is a heavily populated area containing four major cities of Costa Rica located within its boundaries $(\sim 60 \%$ population) along with significant industrial activity. Weather conditions in the valley are mainly influenced by easterly Caribbean trade-winds during the dry season (February-April) and by frequent westerly continental air masses during the wet season (May-October). About $\sim 50 \%$ of GNIP were located within the valley limits. In this region, mean annual precipitation ranges from $\sim 3500 \mathrm{~mm}$ in the highlands to $\sim 2000 \mathrm{~mm}$ within the foothills (Figure 2), and the area experiences 3 - 4 dry months over the summer. This inter-mountainous valley region has an elevation between 1000 and 2500 meters m.a.s.l, with average temperatures that range from $14^{\circ} \mathrm{C}$ to $18^{\circ} \mathrm{C}$. The northern Pacific region of the Nicoya Peninsula receives on average $1500 \mathrm{~mm}$ of precipitation and suffers extensive dry periods that can reach up to 6 months. The Caribbean slope is characterized by a short dry season (1 - 2 months); mean annual precipitation in this region ranges from 4500 to $6000 \mathrm{~mm}$.

\subsection{Historic GNIP Records Analysis}

We analyzed stable isotope records $(n=679)$ of 46 monitoring stations across Costa Rica obtained from the GNIP data base (Table 1, Figure 1). Station altitude ranges from 0 - 3000 m.a.s.l with a mean altitude of 930 m.a.s.l. Sampling frequency was typically monthly except for one station (Monteverde) where event-based sampling was conducted by Rhodes et al. (2006). The entire data set is comprised of three relative short-term sampling campaigns: 1990-1992, 1997-1998, and 20022005. Weighted $\delta^{18} \mathrm{O}$ and $\delta^{2} \mathrm{H}$ means were calculated for 42 stations (87\%). Linear regression analysis of elevation and arithmetic $\delta^{18} \mathrm{O}$ values was conducted considering 18 stations which are unlikely to be influenced by evaporation enrichment: La Selva, Turrialba, Santa María, San Pablo, Santa Lucía, Birrí, Monteverde, Zurquí, Heinz Hoffman, Monte de la Cruz, Pacayas, Fraijanes, Vara Blanca, La Giralda, Sacramento, Paso Llano, Poás, and Irazú. Likewise, linear regression analysis of precipitation amount and $\delta^{18} \mathrm{O}$ values was determined using data from 11 stations which are representative of all four regions: Turrialba, Puriscal, Fraijanes, Orotina, Santa Barbara, Rancho Redondo, Puntarenas, Hacienda Tempisque, and Sacramento.

Spatial analysis was conducted using the Atlas of Costa Rica 2008 database [37]. The natural neighbor procedure in ArcGIS 9.3 was applied to calculate deuterium excess, $\delta^{18} \mathrm{O}$, and $\delta^{2} \mathrm{H}$ interpolations. High station densities within the Central Valley and Nicoya Peninsula may overemphasize the isotopic signature of these regions. Data limitations constrained this and additional analyses. First, the majority of the stations (61\%) generally reported isotopic values over a 1 year period with monthly values ranging from $n=4$ to 28 with a mean of $n=15$. Second, most of the reported stations were located within the Central Valley (the most populated area) and the North Pacific region (Figure 1). Therefore, a considerable gap exists along the Pacific and Caribbean shores, continental divide, and the northern and southern regions (Figure 1). Third, there was an omission of temperature values which prevented calculation of $\delta^{18} \mathrm{O}$ and temperature correlation.

\subsection{HYSPLIT Model Simulations}

To complement the historic GNIP station data analysis, air parcel back trajectories were calculated for particular locations using the hybrid single particle Lagrangian integrated trajectory model (HYSPLIT) [38]. Isotopic values in precipitation from two distinct study locations, Monteverde (continental divide) and Turrialba (Caribbean Slope), were analyzed. Both a depleted and enriched isotopic event during the 2011 and 2012 water years were chosen from these two locations. Single (24 hours) five days back trajectories were calculated using the vertical velocity model calculation method. Trajectory ensembles were done using the GDAS1 meteorological database. Back trajectories were calculated starting at 2300 UTC. Rainfall rate, downward radiation, 


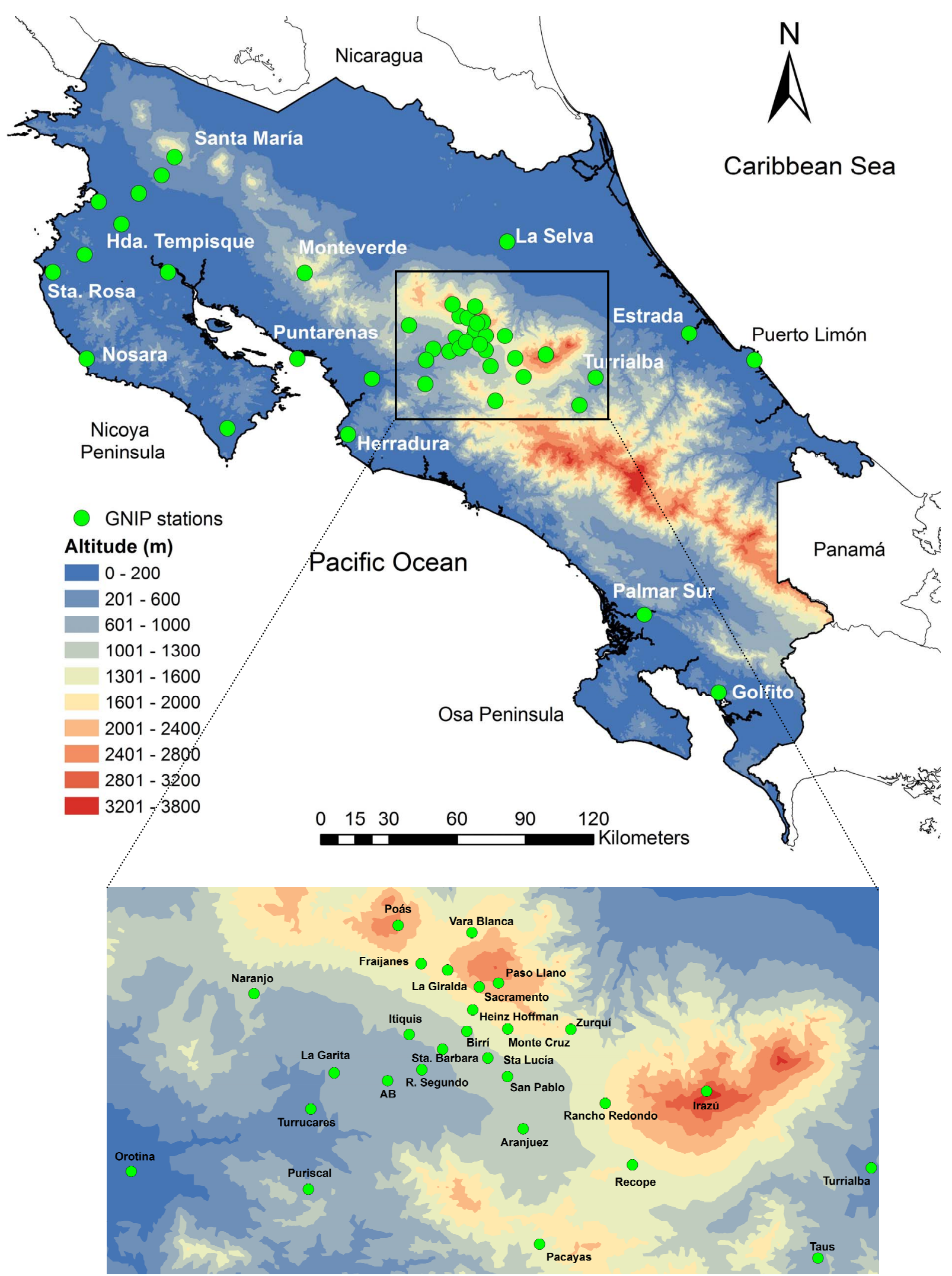

Fgure 1. (Top) Digital elevation map of Costa Rica and location of historic GNIP stations; (bottom) Inset showing Central Valley stations. DEM source: Atlas of Costa Rica 2008 (ITEC); 100 m resolution. 
Table 1. Summary of GNIP stations operated in Costa Rica from 1990 to 2005.

\begin{tabular}{|c|c|c|c|c|c|c|c|c|c|c|c|c|c|c|c|}
\hline \multirow{2}{*}{ Station } & \multirow{2}{*}{$\begin{array}{l}\text { Latitude } \\
\text { (dec.deg) }\end{array}$} & \multirow{2}{*}{$\begin{array}{l}\text { Longitude } \\
\text { (dec.deg) }\end{array}$} & \multirow{2}{*}{$\begin{array}{l}\text { Elevation } \\
\quad(\mathbf{m})\end{array}$} & \multirow{2}{*}{ Climate } & \multirow{2}{*}{$\begin{array}{l}\text { Sampling } \\
\text { period }\end{array}$} & \multirow{2}{*}{$n$} & \multirow{2}{*}{ LMWL } & \multirow{2}{*}{$r^{2}$} & \multirow{2}{*}{$\begin{array}{c}\text { Total } \\
\text { precipitation } \\
(\mathbf{m m})\end{array}$} & \multicolumn{3}{|c|}{ Arithmetic means } & \multicolumn{3}{|c|}{ Weighted means } \\
\hline & & & & & & & & & & $\delta^{18} \mathrm{O}$ & $\delta^{2} \mathbf{H}$ & $d$ & $\delta^{18} \mathrm{O}$ & $\delta^{2} \mathbf{H}$ & $d$ \\
\hline Estrada & 10.0667 & -83.2833 & 6 & Af & $10 / 90-03 / 92$ & 21 & $\delta^{2} \mathrm{H}=7.59 \delta^{18} \mathrm{O}+7.40$ & 0.94 & 1055 & -3.5 & -19.3 & 8.8 & -3.2 & -19.7 & 5.5 \\
\hline La Selva & 10.4314 & -84.0029 & 45 & Aw & $01 / 02-01 / 04$ & 23 & $\delta^{2} \mathrm{H}=8.48 \delta^{18} \mathrm{O}+14.03$ & 0.99 & 4304 & -1.8 & -17.3 & 12.3 & -3.8 & -20.0 & 10.4 \\
\hline Taus & 9.7833 & -83.7167 & 900 & $\mathrm{Cbf}$ & $01 / 90-12 / 90$ & 8 & $\delta^{2} \mathrm{H}=7.97 \delta^{18} \mathrm{O}+11.10$ & 0.97 & 4256 & -7.1 & -45.5 & 11.3 & -7.2 & -45.8 & 11.5 \\
\hline Turrialba & 9.8913 & -83.6529 & 604 & Aw & 01/04-02/02 & 24 & $\delta^{2} \mathrm{H}=8.62 \delta^{18} \mathrm{O}+16.53$ & 0.99 & 3177 & -5.0 & -26.4 & 13.4 & -5.2 & -30.2 & 11.1 \\
\hline $\mathrm{AB}$ & 9.9958 & -84.2319 & 860 & Aw & $12 / 03-10 / 04$ & 12 & $\delta^{2} \mathrm{H}=7.91 \delta^{18} \mathrm{O}+10.11$ & 0.99 & 2017 & -6.7 & -42.8 & 10.7 & -7.3 & -49.1 & 9.5 \\
\hline Aranjuez & 9.9380 & -84.0696 & 1191 & Aw & $02 / 02-01 / 04$ & 19 & $\delta^{2} \mathrm{H}=8.16 \delta^{18} \mathrm{O}+12.11$ & 0.99 & 1910 & -8.3 & -55.6 & 10.8 & -9.3 & -64.5 & 9.8 \\
\hline Birrí & 10.0550 & -84.1370 & 1318 & Aw & $12 / 03-10 / 04$ & 12 & $\delta^{2} \mathrm{H}=8.40 \delta^{18} \mathrm{O}+14.20$ & 0.99 & 2916 & -6.6 & -41.2 & 11.6 & -7.3 & -49.0 & 9.4 \\
\hline Fraijanes & 10.1359 & -84.1917 & 1737 & Aw & $01 / 02-01 / 12$ & 24 & $\delta^{2} \mathrm{H}=8.47 \delta^{18} \mathrm{O}+16.58$ & 0.99 & 3506 & -6.8 & -40.9 & 13.4 & -7.8 & -51.4 & 10.7 \\
\hline $\begin{array}{l}\text { Heinz } \\
\text { Hoffman }\end{array}$ & 10.0807 & -84.1299 & 1650 & Aw & $12 / 03-10 / 04$ & 12 & $\delta^{2} \mathrm{H}=8.16 \delta^{18} \mathrm{O}+12.45$ & 0.99 & 2940 & -6.6 & -41.1 & 11.4 & -7.1 & -46.9 & 9.8 \\
\hline Irazú & 9.9833 & -83.8500 & 3000 & $\mathrm{Cbf}$ & $01 / 90-12 / 90$ & 8 & $\delta^{2} \mathrm{H}=7.85 \delta^{18} \mathrm{O}+8.91$ & 0.98 & N.A. & -10.1 & -70.4 & 10.4 & N.A. & N.A. & N.A. \\
\hline Itiquis & 10.0511 & -84.2061 & 1110 & Aw & $10 / 03-9 / 04$ & 11 & $\delta^{2} \mathrm{H}=7.93 \delta^{18} \mathrm{O}+10.71$ & 0.99 & 3026 & -7.1 & -45.8 & 11.2 & -8.4 & -56.7 & 10.3 \\
\hline La Garita & 10.0052 & -84.2957 & 760 & Aw & $10 / 03-9 / 04$ & 12 & $\delta^{2} \mathrm{H}=7.97 \delta^{18} \mathrm{O}+10.99$ & 0.99 & 2349 & -6.5 & -40.8 & 11.2 & -7.1 & -47.6 & 9.1 \\
\hline La Giralda & 10.1282 & -84.1600 & 2014 & $\mathrm{Cfa}$ & $01 / 02-12 / 03$ & 20 & $\delta^{2} \mathrm{H}=8.49 \delta^{18} \mathrm{O}+16.71$ & 0.99 & 2708 & -6.8 & -41.3 & 13.3 & -7.3 & -47.9 & 10.6 \\
\hline $\begin{array}{l}\text { Monte } \\
\text { de la Cruz }\end{array}$ & 10.0577 & -84.0879 & 1700 & Aw & $10 / 03-09 / 04$ & 13 & $\delta^{2} \mathrm{H}=8.24 \delta^{18} \mathrm{O}+12.80$ & 0.99 & 2189 & -7.3 & -47.1 & 11.0 & -9.2 & -62.9 & 11.1 \\
\hline Naranjo & 10.1001 & -84.3917 & 1051 & Aw & $01 / 02-12 / 03$ & 15 & $\delta^{2} \mathrm{H}=7.98 \delta^{18} \mathrm{O}+9.80$ & 0.99 & 2064 & -7.6 & -51.0 & 10.0 & -11.1 & -76.8 & 12.2 \\
\hline Paso Llano & 10.1128 & -84.0991 & 2397 & $\mathrm{Cfa}$ & $12 / 03-10 / 04$ & 12 & $\delta^{2} \mathrm{H}=8.40 \delta^{18} \mathrm{O}+16.27$ & 0.99 & 4131 & -6.9 & -41.8 & 13.5 & -6.6 & -41.6 & 11.1 \\
\hline Poás & 10.1818 & -84.2193 & 2500 & $\mathrm{Cbf}$ & $12 / 97-06 / 99$ & 14 & $\delta^{2} \mathrm{H}=8.12 \delta^{18} \mathrm{O}+14.52$ & 0.99 & N.A. & -7.7 & -48.3 & 13.6 & N.A. & N.A. & N.A. \\
\hline Puriscal & 9.8656 & -84.3267 & 836 & Aw & 01/02-01/04 & 22 & $\delta^{2} \mathrm{H}=8.03 \delta^{18} \mathrm{O}+12.21$ & 0.99 & 2694 & -6.8 & -43.4 & 11.0 & -8.3 & -55.9 & 10.5 \\
\hline $\begin{array}{l}\text { Rancho } \\
\text { Redondo }\end{array}$ & 9.9686 & -83.9713 & 1662 & Aw & $02 / 02-01 / 04$ & 21 & $\delta^{2} \mathrm{H}=8.20 \delta^{18} \mathrm{O}+13.20$ & 0.98 & 2435 & -8.2 & -53.7 & 11.5 & -9.9 & -68.7 & 10.7 \\
\hline Recope & 9.8949 & -83.9388 & 1563 & Aw & $02 / 02-01 / 04$ & 19 & $\delta^{2} \mathrm{H}=8.44 \delta^{18} \mathrm{O}+15.45$ & 0.99 & 1179 & -8.4 & -55.3 & 11.8 & -10.7 & -73.3 & 12.0 \\
\hline $\begin{array}{l}\text { Río } \\
\text { Segundo }\end{array}$ & 10.0089 & -84.1908 & 980 & Aw & $12 / 03-10 / 04$ & 11 & $\delta^{2} \mathrm{H}=8.59 \delta^{18} \mathrm{O}+16.11$ & 0.99 & 2092 & -7.0 & -43.7 & 13.6 & -8.1 & -54.5 & 10.3 \\
\hline Sacramento & 10.1081 & -84.1221 & 2260 & $\mathrm{Cbf}$ & $\begin{array}{l}01 / 90-12 / 90 \\
01 / 03-12 / 04\end{array}$ & 21 & $\delta^{2} \mathrm{H}=8.39 \delta^{18} \mathrm{O}+13.62$ & 0.99 & 2577.5 & -7.4 & -47.3 & 11.5 & -8.6 & -57.7 & 11.5 \\
\hline San Pablo & 10.0008 & -84.0882 & 1215 & Aw & $12 / 03-10 / 04$ & 12 & $\delta^{2} \mathrm{H}=8.30 \delta^{18} \mathrm{O}+13.19$ & 0.99 & 2051 & -6.6 & -41.4 & 11.2 & -7.6 & -51.4 & 9.4 \\
\hline $\begin{array}{l}\text { Santa } \\
\text { Barbara }\end{array}$ & 10.0334 & -84.1660 & 1102 & Aw & 02/02-01/04 & 21 & $\delta^{2} \mathrm{H}=8.19 \delta^{18} \mathrm{O}+12.01$ & 0.99 & 2522 & -7.5 & -49.4 & 10.3 & -9.3 & -63.6 & 10.8 \\
\hline Santa Lucía & 10.0230 & -84.1118 & 1251 & Aw & $01 / 02-12 / 03$ & 18 & $\delta^{2} \mathrm{H}=8.19 \delta^{18} \mathrm{O}+12.01$ & 0.99 & 1793 & -6.4 & -40.1 & 10.8 & -10.8 & -74.8 & 12.0 \\
\hline Turrucares & 9.9617 & -84.3237 & 642 & Aw & $12 / 03-10 / 04$ & 11 & $\delta^{2} \mathrm{H}=7.93 \delta^{18} \mathrm{O}+9.66$ & 0.99 & 1945 & -6.5 & -41.7 & 10.1 & -7.6 & -51.2 & 9.5 \\
\hline Vara Blanca & 10.1732 & -84.1310 & 1845 & Aw & $12 / 03-10 / 04$ & 12 & $\delta^{2} \mathrm{H}=8.40 \delta^{18} \mathrm{O}+14.84$ & 0.99 & 4579 & -6.0 & -36.0 & 12.4 & -5.2 & -31.5 & 8.2 \\
\hline Zurquí & 10.0573 & -84.0124 & 1548 & Aw & $03 / 02-05 / 04$ & 28 & $\delta^{2} \mathrm{H}=8.57 \delta^{18} \mathrm{O}+17.53$ & 0.99 & 5074 & -5.6 & -30.1 & 14.4 & -6.0 & -30.4 & 17.4 \\
\hline Capulin & 10.6228 & -85.4611 & 125 & Aw & $11 / 97-05 / 98$ & 8 & $\delta^{2} \mathrm{H}=7.79 \delta^{18} \mathrm{O}+6.82$ & 0.99 & N.A. & -8.4 & -59.0 & 8.6 & N.A. & N.A. & N.A. \\
\hline Cartagena & 10.3800 & -85.6750 & 63 & Aw & 01/91-12/91 & 7 & $\delta^{2} \mathrm{H}=7.83 \delta^{18} \mathrm{O}+1.95$ & 0.97 & 1010 & -5.7 & -42.5 & 2.9 & -6.3 & -47.1 & 3.5 \\
\hline
\end{tabular}




\begin{tabular}{|c|c|c|c|c|c|c|c|c|c|c|c|c|c|c|c|}
\hline Cóbano & 9.6900 & -85.1100 & 160 & Aw & $01 / 90-12 / 90$ & 8 & $\delta^{2} \mathrm{H}=8.42 \delta^{18} \mathrm{O}+13.57$ & 0.98 & 2400 & -6.5 & -41.4 & 10.8 & -7.1 & -46.3 & 10.5 \\
\hline $\begin{array}{l}\text { Est. } \\
\text { Ecológica }\end{array}$ & 10.6944 & -85.3706 & 340 & Aw & $11 / 97-05 / 98$ & 7 & $\delta^{2} \mathrm{H}=8.03 \delta^{18} \mathrm{O}+8.77$ & 0.95 & N.A. & -8.2 & -57.4 & 8.5 & N.A. & N.A. & N.A. \\
\hline $\begin{array}{l}\text { Hacienda } \\
\text { Tempisque }\end{array}$ & 10.5000 & -85.5300 & 22 & Aw & 05/90-07/90 & 13 & $\delta^{2} \mathrm{H}=7.81 \delta^{18} \mathrm{O}+5.52$ & 0.92 & 897 & -5.2 & -35.1 & 6.5 & -6.2 & -42.3 & 7.2 \\
\hline Monte Galan & 10.5900 & -85.6200 & 60 & Aw & $01 / 90-12 / 90$ & 8 & $\delta^{2} \mathrm{H}=6.06 \delta^{18} \mathrm{O}-2.69$ & 0.91 & 1185 & -5.4 & -35.4 & 7.8 & -6.0 & -37.4 & 10.6 \\
\hline Nosara & 9.9667 & -85.6667 & 15 & Aw & $05 / 90-06 / 91$ & 14 & $\delta^{2} \mathrm{H}=8.43 \delta^{18} \mathrm{O}+10.89$ & 0.92 & 1591 & -6.3 & -42.5 & 8.2 & -6.3 & -43.2 & 7.6 \\
\hline $\begin{array}{l}\text { Puerto } \\
\text { Humo }\end{array}$ & 10.3100 & -85.3450 & 10 & Aw & $01 / 90-12 / 90$ & 9 & $\delta^{2} \mathrm{H}=8.60 \delta^{18} \mathrm{O}+14.50$ & 0.99 & 1374 & -6.6 & -42.5 & 10.5 & -6.5 & -40.8 & 10.8 \\
\hline Santa María & 10.7667 & -85.3200 & 825 & Am & $\begin{array}{c}01 / 90-12 / 92 \\
01 / 97-12 / 98\end{array}$ & 26 & $\delta^{2} \mathrm{H}=8.04 \delta^{18} \mathrm{O}+9.67$ & 0.99 & 1901 & -5.5 & -34.8 & 9.5 & -5.9 & -38.7 & 8.6 \\
\hline Santa Rosa & 10.3100 & -85.8000 & 25 & Aw & $01 / 90-12 / 90$ & 4 & $\delta^{2} \mathrm{H}=8.24 \delta^{18} \mathrm{O}+13.30$ & 0.99 & 992 & -9.2 & -62.1 & 11.1 & -8.6 & -57.5 & 11.5 \\
\hline Herradura & 9.6667 & -84.6333 & 3 & $\mathrm{Am}$ & $01 / 90-12 / 90$ & 8 & $\delta^{2} \mathrm{H}=8.18 \delta^{18} \mathrm{O}+12.32$ & 0.99 & 3082 & -6.9 & -44.5 & 11.1 & -7.3 & -47.2 & 11.0 \\
\hline Orotina & 9.8870 & -84.5387 & 168 & Aw & 03/02-01/04 & 19 & $\delta^{2} \mathrm{H}=7.93 \delta^{18} \mathrm{O}+9.70$ & 0.99 & 2256 & -6.3 & -40.1 & 10.1 & -8.5 & -56.1 & 11.7 \\
\hline Palmar Sur & 8.9500 & -83.4600 & 16 & $\mathrm{Am}$ & $01 / 90-12 / 90$ & 8 & $\delta^{2} \mathrm{H}=8.50 \delta^{18} \mathrm{O}+14.17$ & 0.99 & 3293 & -7.6 & -50.1 & 10.4 & -7.9 & -52.9 & 10.0 \\
\hline Puntarenas & 9.9667 & -84.8333 & 3 & Aw & $\begin{array}{l}01 / 93-12 / 93 \\
01 / 03-12 / 04\end{array}$ & 15 & $\delta^{2} \mathrm{H}=8.10 \delta^{18} \mathrm{O}+12.00$ & 0.99 & 1443.35 & -7.2 & -46.7 & 11.3 & -8.9 & -59.7 & 11.6 \\
\hline Monteverde & 10.3067 & -84.8047 & 1460 & $\mathrm{Am}$ & 06/03-03/05 & 42 & $\delta^{2} \mathrm{H}=8.60 \delta^{18} \mathrm{O}+14.28$ & 0.99 & N.A. & -6.1 & -37.9 & 10.7 & N.A. & N.A. & N.A. \\
\hline Mean & & & & & & & & & & -6.7 & -43.2 & 10.8 & -7.4 & -49.0 & 10.3 \\
\hline Max & & & & & & & & & & -1.8 & -17.3 & 14.4 & -3.2 & -19.7 & 17.4 \\
\hline Min & & & & & & & & & & -10.1 & -70.4 & 2.9 & -11.1 & -76.8 & 3.5 \\
\hline
\end{tabular}

Köppen-Geiger code Aw = tropical wet and dry; Am = tropical trade-wind littoral; Af = tropical rainforest; Cbf = tropical highland; Cfa = humid; $\mathrm{Cwb}=$ tropical highland. $\mathrm{N} . \mathrm{A} .=$ not available.

relative humidity, and elevation of the mixing layer depth were also computed.

\subsection{Stable Isotope Analysis}

Precipitation was collected on event-basis. Monteverde sampling was carried out during July, August, and September of 2011. Turrialba's sampling period used in this study was from September to December, 2012. The collector for individual events consisted of a $10.16 \mathrm{~cm}$ plastic funnel coupled with a metal filter mesh (i.e., to prevent external contamination). The funnel was connected to $4 \mathrm{~L}$ high density polyethylene (HDPE) container. An approximately $3 \mathrm{~cm}$ mineral oil layer was added to prevent fractionation according with standard sampling protocols. Later, mineral oil was separated using a $250-500 \mathrm{~mL}$ separatory funnel. Samples were stored upside down in a HDPE bottles with conic and polyseal inserts and parafilm seals until analysis. Stable isotope analyses were conducted at the Chemistry School of the National University (Heredia, Costa Rica) and the Idaho Stable Isotope Laboratory (Moscow, Idaho) using a Cavity Ring Down
Spectroscopy (CRDS) water isotope analyzer L2120-I (Picarro, CA) and L1120-i (Picarro, CA), respectively. Ratios of $\delta^{18} \mathrm{O} / \delta^{16} \mathrm{O}$ and $\delta^{2} \mathrm{H} / \delta^{1} \mathrm{H}$ are expressed in delta units (\%o, parts per mil) relative to Vienna Standard Mean Ocean Water (V-SMOW). The current analytical analyzers precision is $0.1 \%$ o $\delta^{18} \mathrm{O} / \delta^{16} \mathrm{O}$ and $0.5 \%$ o $\delta^{2} \mathrm{H} / \delta^{1} \mathrm{H}$.

\section{Results and Discussion}

\subsection{Precipitation}

Precipitation variability in Costa Rica reflects regional and local complex dynamics. Figure 2 shows mean annual precipitation throughout Costa Rica and precipitation amounts recorded during isotopic sampling campaigns. GNIP precipitation records are consistent with the Atlas of Costa Rica reported values and ranged from $5074 \mathrm{~mm}$ (Zurquí) to $897 \mathrm{~mm}$ (Hacienda Tempisque) with a mean of $2479 \mathrm{~mm}$ (Table 1, Figure 2). The lowlands of the Nicoya Peninsula is the region with the lowest precipitation regime $(<1500 \mathrm{~mm})$ while the Talamanca Range and northeastern Caribbean lowlands received the greatest amount, between 4500 - $8000 \mathrm{~mm}$ 


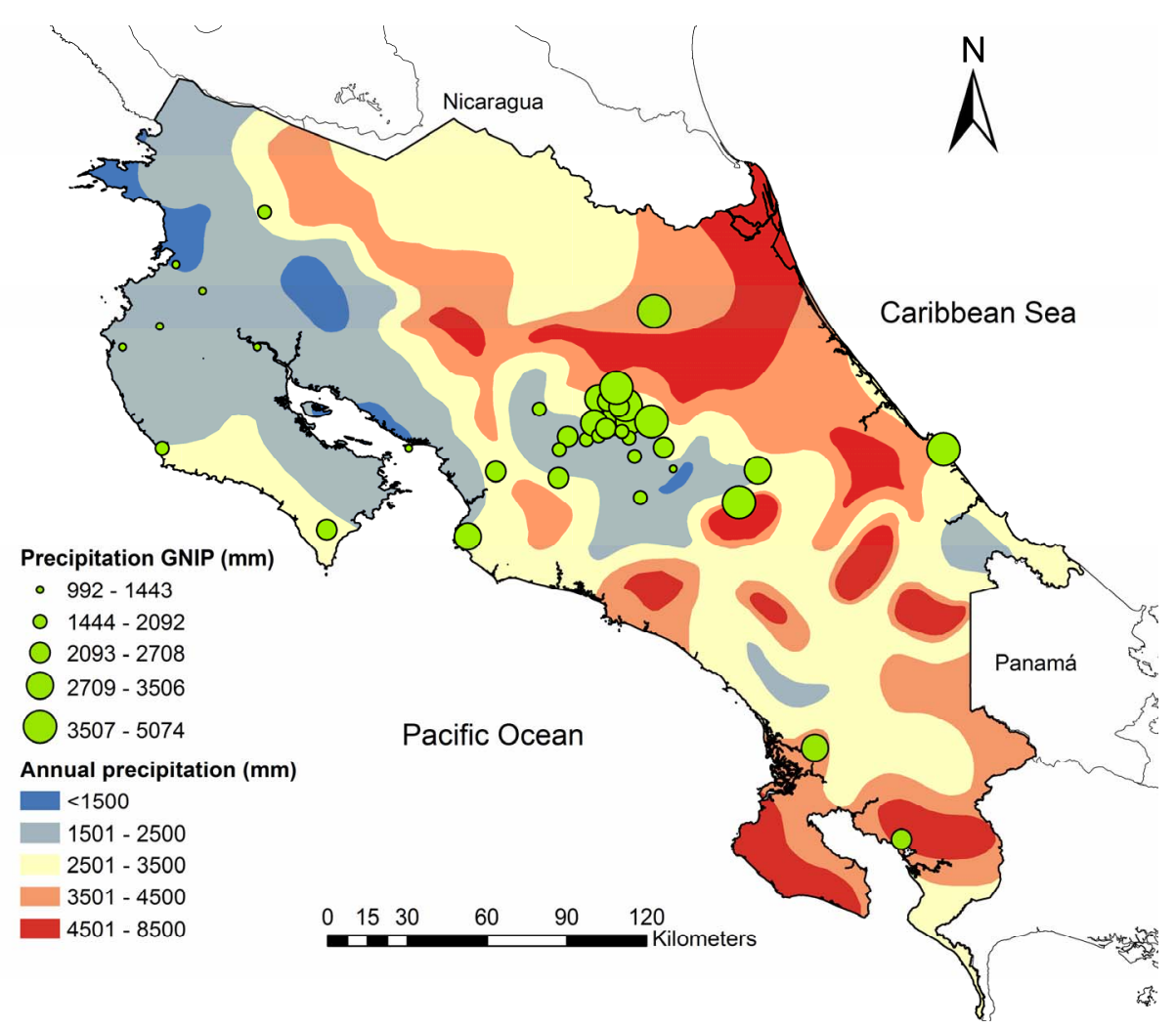

Figure 2. Mean annual precipitation throughout Costa Rica with GNIP stations showing precipitation recorded during sampling periods.

annually. These precipitation records provide a reliable basis for further stable isotope spatial analysis.

\section{2. $\delta^{18} \mathrm{O}$ and $\delta^{2} \mathrm{H}$ Isotopic Variations}

Stable isotope signatures in precipitation across Costa Rica were variable during the sampling campaigns. $\delta^{18} \mathrm{O}$ composition ranged from $-1.8 \%$ to $-10.1 \%$ with an arithmetic mean of $-6.7 \pm 1.4(\%)$ (Figure 3, Table 1). Weighted $\delta^{18} \mathrm{O}$ values range from $-3.2 \%$ to $-11.1 \%$ with a mean of $-7.4 \pm 1.8(\%$ ) (Table 1). Deuterium composition varied from $-17.3 \%$ to $-70.4 \%$ with an arithmetic mean of $-43.2 \pm 10.3(\%$ ) (Figure 3, Table 1). Weighted $\delta^{2} \mathrm{H}$ values ranged from $-19.7 \%$ o to $-76.8 \%$ o with a mean of $-49.0 \pm 13.7(\%$ ) (Table 1). Figure 4 shows the regional water meteoric lines for each of the four main regions for the GNIP Stations (Nicoya Peninsula, Pacific Coast, Caribbean Slope, and Central Valley). The best fit straight line to the data for the Nicoya Peninsula yields an arithmetic water line equation of $\delta^{2} \mathrm{H}=$ $7.84 \delta^{18} \mathrm{O}+7.60$ (Figure 4), whereas the weighted meteoric water line yields a distinct best fit straight line equation of $\delta^{2} \mathrm{H}=6.65 \delta^{18} \mathrm{O}-0.13$ (Figure 4), likely resulting from small precipitation amounts. For the Pacific Coast, the best fit straight line to precipitation-weighted annual means is $\delta^{2} \mathrm{H}=7.60 \delta^{18} \mathrm{O}+7.95$ (Figure 4), which is consistent with the Global Meteoric Water Line (GMWL) of $\delta^{2} \mathrm{H}=8 \delta^{18} \mathrm{O}+10$ [39]. Interestingly, Pacific Coast stations are close to equilibrium conditions rather than exhibiting evaporation enrichment. The Caribbean Slope precipitation-weighted water line $\delta^{2} \mathrm{H}=6.98 \delta^{18} \mathrm{O}+4.97$ (Figure 4) reflects orographic distillation of Caribbean-sourced moisture. Overall, the best fit line to the precipitation-weighted data for all regions combined yields a water line of $\delta^{2} \mathrm{H}=7.61 \delta^{18} \mathrm{O}+7.40\left(r^{2}=0.98\right)$ which corresponds closely to the GMWL (Figure 5). Enriched values above the annual mean of $-6.7 \%$ o $\delta^{18} \mathrm{O}$ were often observed during January to March. The greater variation occurred in May and June when the ITCZ starts its migration over Costa Rica. Towards the middle of the wet season (August, September, and October) $\delta^{18} \mathrm{O}$ variation appears to decrease due to increase in precipitation amounts. The intensification of northeast trade winds in December produced a decrease in precipitation, and consequently, enrichment in isotopic values (Figure 5).

\subsection{The Altitude Effect}

The variability in isotopic composition of precipitation with elevation is not consistent across the GNIP stations indicating a complex system. Figure 6 shows three lon- 

An Analysis of Historic GNIP Records
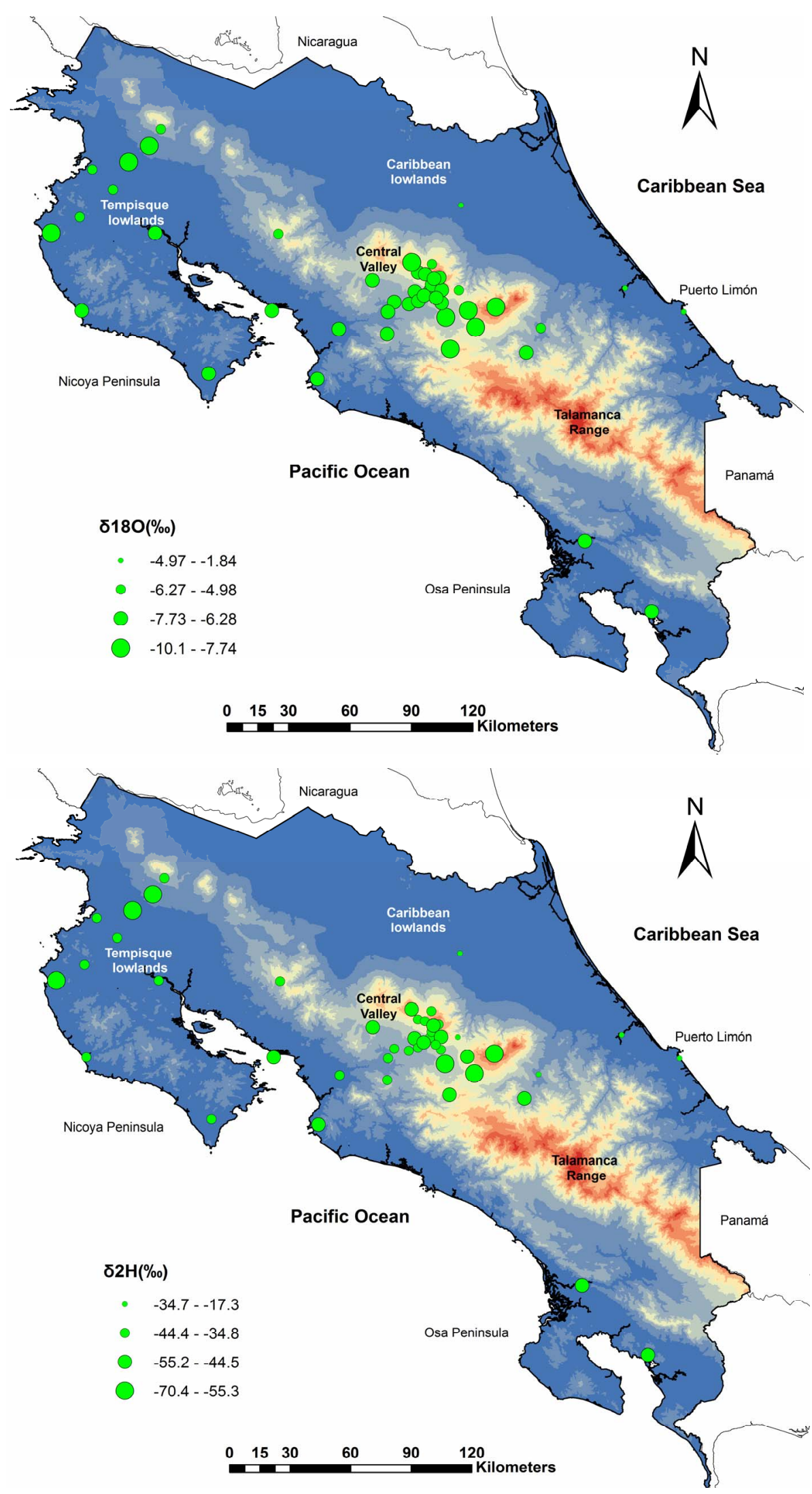

Figure 3. Spatial variations of $\delta^{18} \mathrm{O}$ (top) and $\delta^{2} \mathrm{H}$ (bottom) arithmetic means. 

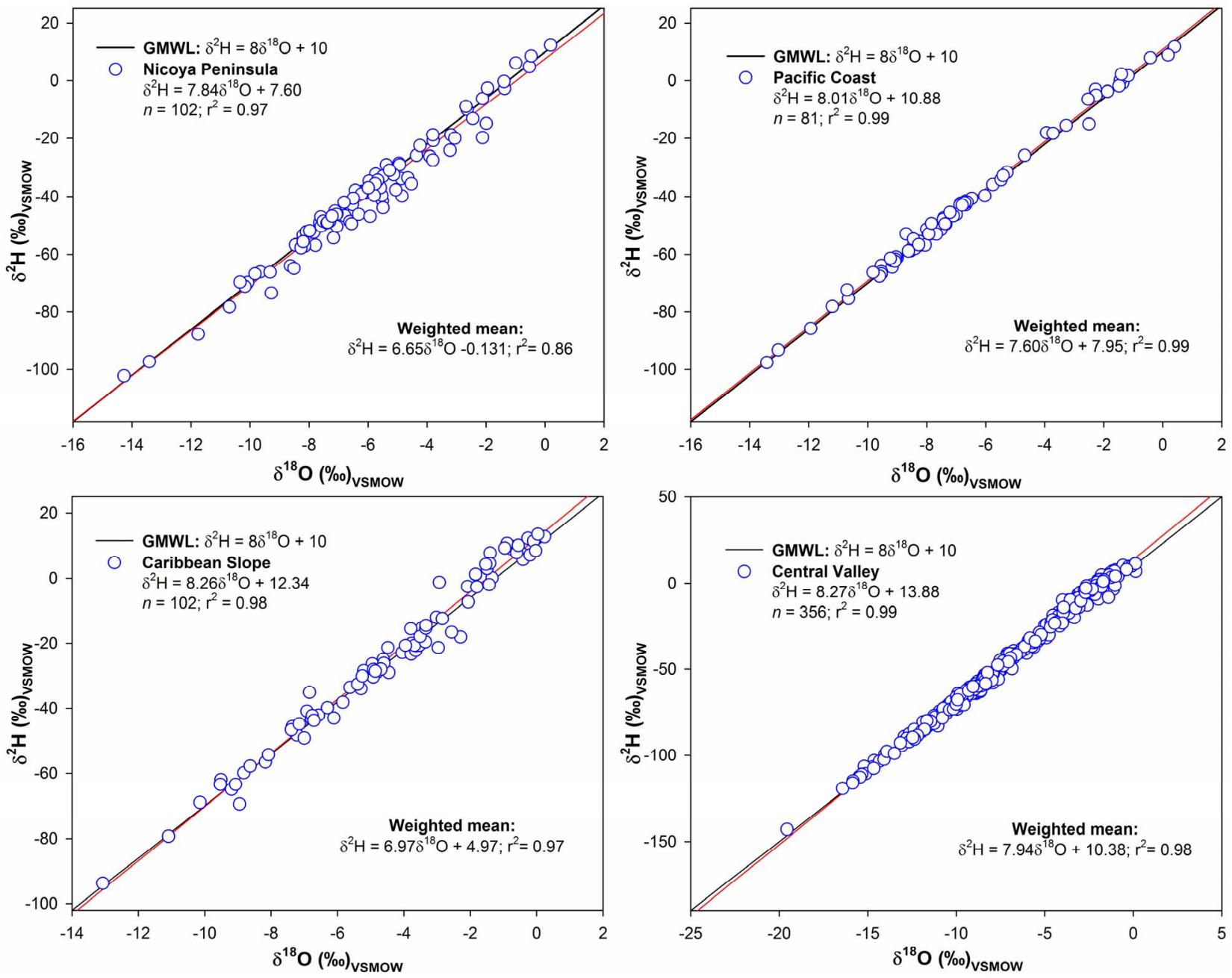

Figure 4. Meteoric water lines for four major geographic regions of Costa Rica.

gitudinal transects. For the Caribbean slope, there is an evident orographic distillation effect from Puerto Limón (0 m.a.s.1.) to Taus ( 900 m.a.s.l.) of $-3.5 \% \delta^{18} \mathrm{O} / \mathrm{km}$. The $\sim 75 \mathrm{~km}$ Pacific slope transect from Herradura (3 m.a.s.1.) to Paso Llano (2397 m.a.s.1.) represents a more complex system. For instance, a rainout effect is observed up to the Santa Bárbara station (1102 m.a.s.l.) (Figure 6). However, at the two higher elevation stations (Birrí and Heinz Hoffman) isotopic compositions are more enriched, suggesting another moisture source. A similar phenomena is also observed along the Nicoya Peninsula transect of $\sim 66 \mathrm{~km}$ from the coast to the Rincón de la Vieja volcano (Figure 6).

Considering the 18 stations mainly from the Central Valley cluster, the correlation of elevation with arithmetic mean annual $\delta^{18} \mathrm{O}$ is moderately strong $\left(r^{2}=0.77\right)$. The best fit straight line has a slope of $-0.2 \% 0 \delta^{18} \mathrm{O}$ per $100 \mathrm{~m}$, which is close to the range of $0.1 \%$ and $0.36 \%$ reported by Leibungdgut et al. (2009) [40]. Since the altitude effect is the combined result of the temperature effect and moisture depletion by adiabatic cooling, a temperature effect can be calculated by knowing the atmospheric lapse rate. Lachniet and Paterson (2002) [27] reported a lapse rate of $-5.4^{\circ} \mathrm{C} / \mathrm{km}$ for the San José area. Therefore, an altitude effect of $-0.2 \% / \mathrm{km}$ corresponds to $-0.37 \% \circ /{ }^{\circ} \mathrm{C}$, in agreement with temperature effects reported in the pioneer work done by Dansgaard [23].

\subsection{The Amount Effect and d-Excess Isoscape}

One important factor controlling isotopic variations in the tropics is the "amount effect" $[23,24,41]$. This effect has been attributed to three main factors according to Scholl et al. (2009) [42]: 1) the isotopic composition of the condensation in a cloud decreases as cooling and "rainout" occurs; 2) smaller raindrops equilibrate to a larger degree with the water vapor and temperature conditions below the cloud; and 3) small raindrops evaporate more than larger raindrops on their way to the land 

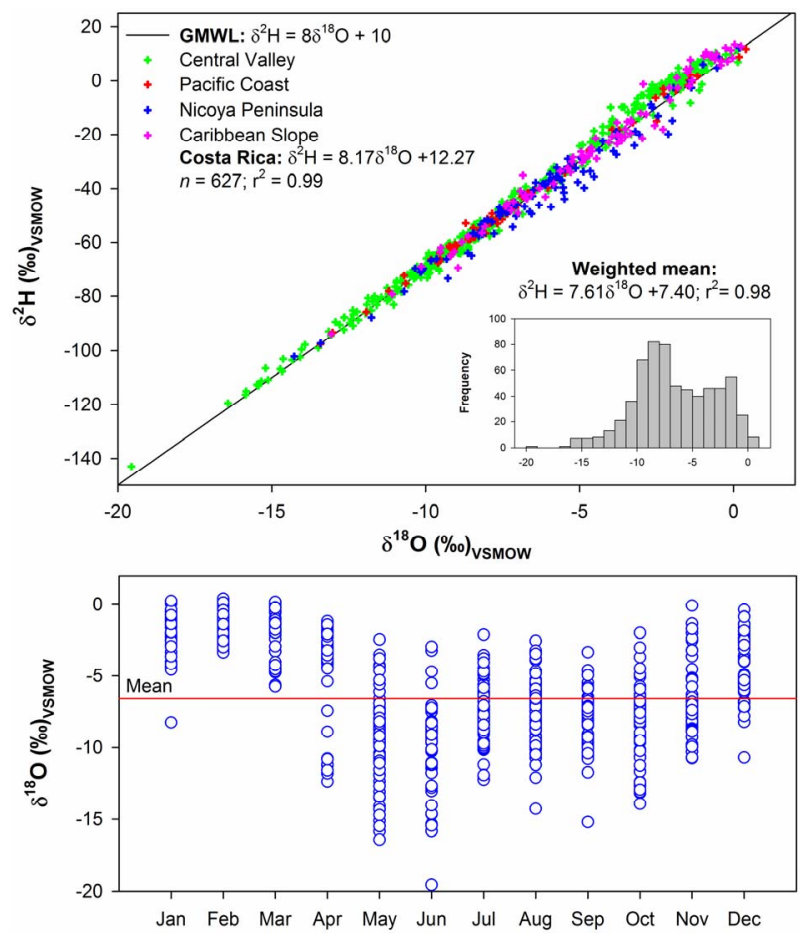

Figure 5. (Top) Meteoric water line for Costa Rica. Contributions from all four regions are color coded. Inset: distribution of $\delta^{18} \mathrm{O}$ values; (Bottom) Temporal variation of $\delta^{8} \mathrm{O}$ values.

surface. The slope of the best fit straight line to precipitation amount and $\delta^{18} \mathrm{O}$ from 11 stations, which are representative of the four main regions described previously, was found to be $-1.6 \%$ per $100 \mathrm{~mm}$ decrease in rainfall $\left(r^{2}=0.57\right)$. This effect is clearly present during the times of lowest precipitation (January to April).

The "amount effect" was also observed in the $d$-excess isoscape presented in Figure 7. D-excess composition ranged from $2.9 \%$ to $14.4 \%$ with an arithmetic mean of $10.8 \pm 2.0(\%)$ (Table 1, Figure 7). Weighted $d$-excess values range from $3.5 \%$ to $17.4 \%$ with a mean of $10.3 \pm$ $2.1(\%)$ (Table 1, Figure 7). Low d-excess $(\sim 3 \%)$ is predominantly present within the Nicoya Peninsula and Caribbean lowlands and may also be a common phenomena across the northern lowlands and Nicaragua trough. High $d$-excess $(>17 \%$ ) was found in the dense forested areas east of the Central Valley (Zurquí and Irazú mountain range) and the Pacific slope of the Monteverde tropical cloud forest (Figure 7), which may indicate successive evaporation and precipitation cycles. Based on d-excess values, Rhodes et al. (2006) [43] concluded that moisture flux from evaporation is an important input to the region during the transitional and dry seasons when trade winds from the Caribbean slope dominate. Therefore, it appears that intense moisture recycling processes govern isotopic variations within the
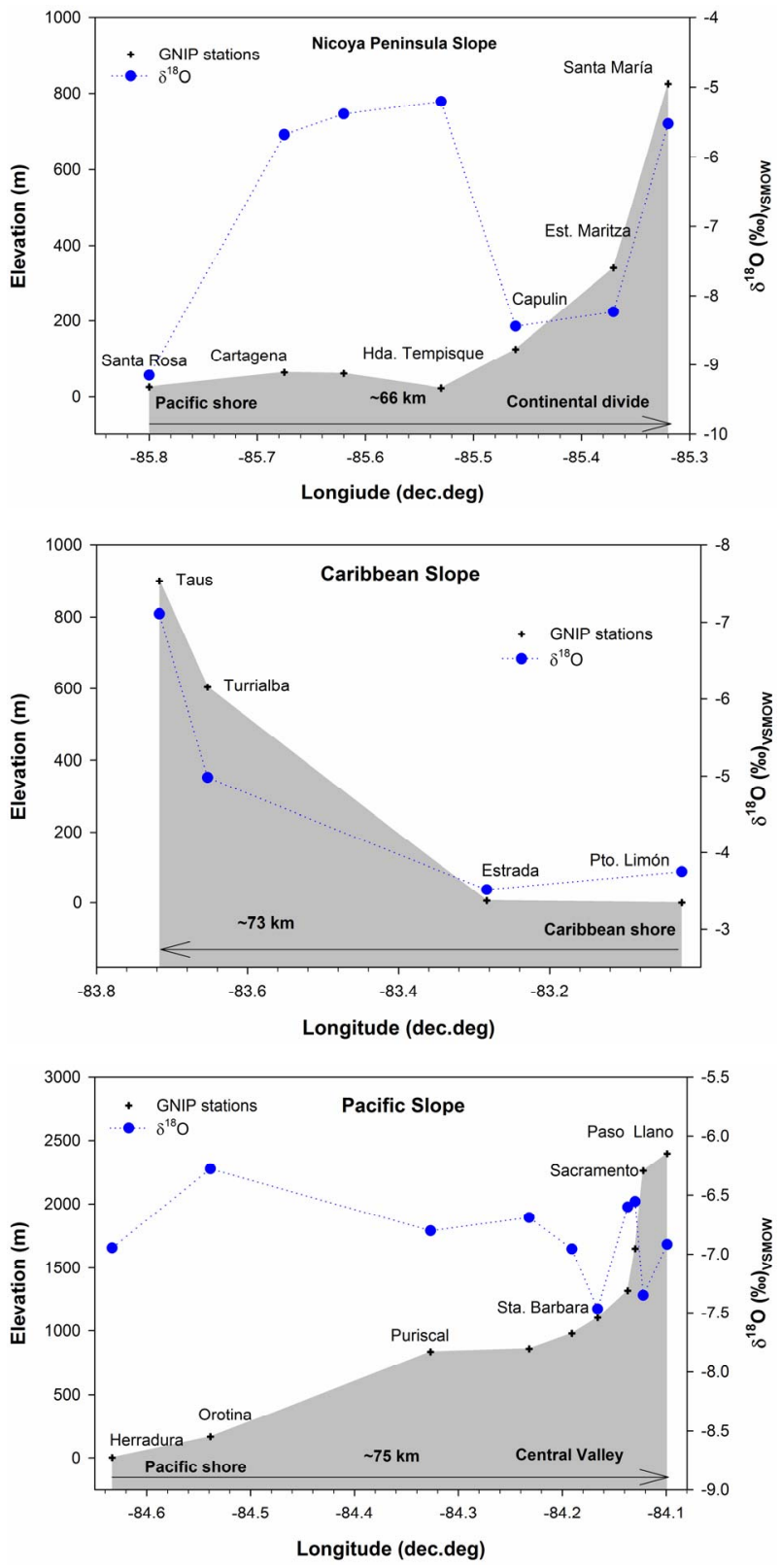

Figure 6. Longitudinal elevation and isotopic transects of Nicoya Peninsula, Pacific and Caribbean slopes.

inter-mountainous regions of Costa Rica.

\subsection{Air Mass Back Trajectories and Precipitation Events}

Figure 8 shows daily variation in isotopic composition in precipitation at Monteverde and Turrialba. For Monteverde, a remarkable depletion in $\delta^{18} \mathrm{O}$ was observed on $7 / 18 / 2011$ and an enriched event was sampled on 7/26/ 2011 (Figure 8). For Turrialba, sampling coincided with the wet to dry season transition, but sampling events for the purposes of the air mass trajectories were selected 


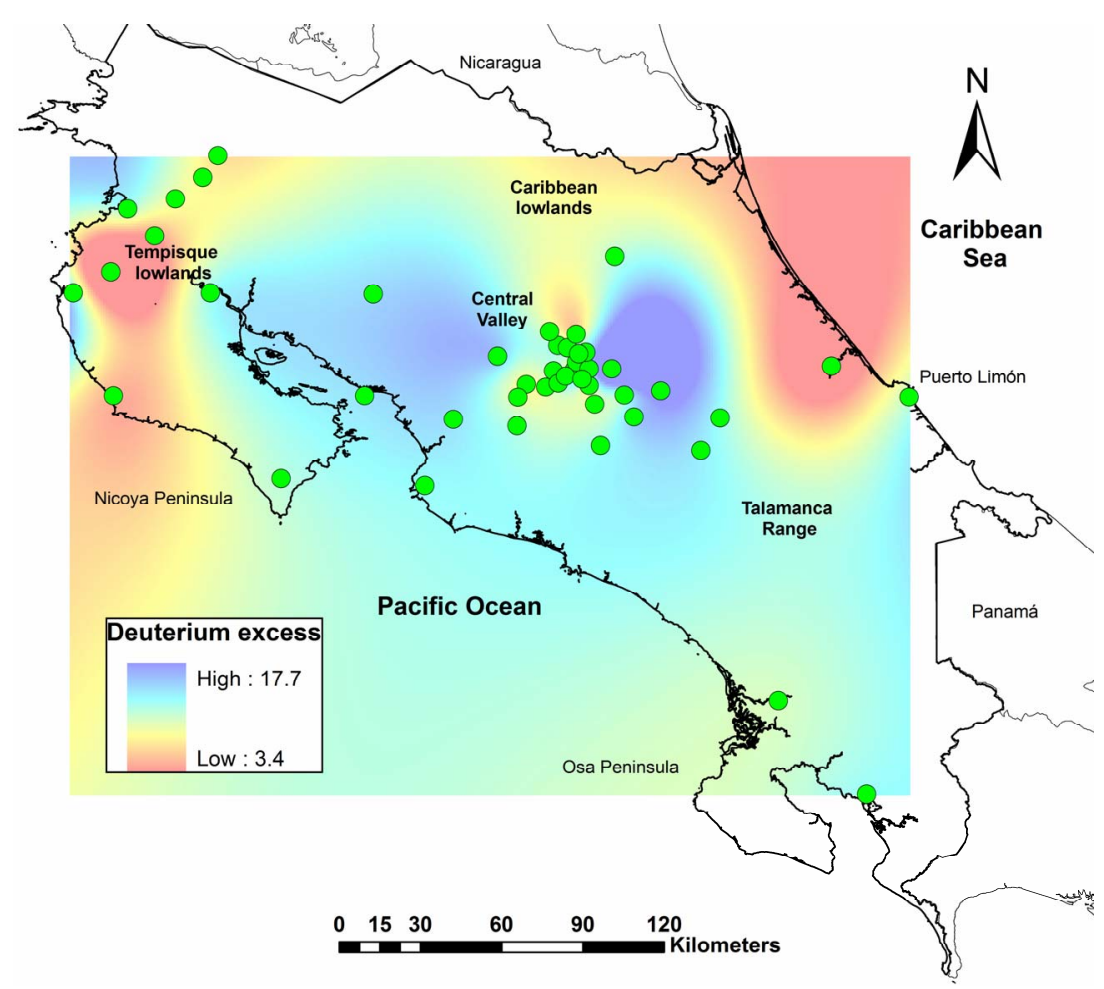

Figure 7. Preliminary d-excess isoscape for Costa Rica.
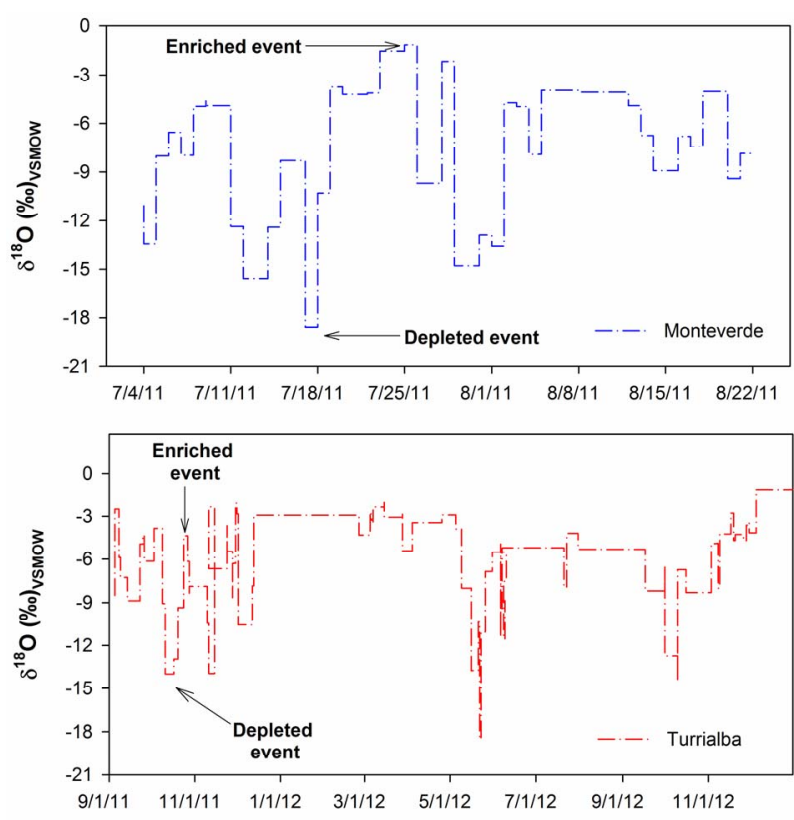

Figure 8. Daily $\delta^{18} \mathrm{O}$ variations in precipitation at Monteverde and Turrialba stations.

during the wet and transitional seasons (September-December, 2011). Considerably depleted and enriched $\delta^{18} \mathrm{O}$ values were recorded on 10/17/2011 and 10/27/2011, respectively (Figure 8). Figure 9 shows air mass back trajectories of five days prior to the depleted or enriched precipitation event coupled with rainfall intensities. HYSPLIT simulated trajectories indicate that enriched isotopic compositions both in Turrialba and Monteverde correspond to central Caribbean-sourced moisture and small rainfall intensities (Figures 9(a) and (b)). Depleted events appear to correspond to high rainfall amounts despite the parental origin of the moisture. In the case of Turrialba, the depleted $\delta^{18} \mathrm{O}$ event was related to continental air masses arriving over Costa Rica from the southwest, producing convective precipitation as they travel along and over the Pacific Coast. Air masses intrusions from the Pacific Ocean are typically observed during the wet season and are less frequent when the wet to dry season transition begins in November. The depleted precipitation sampled in Monteverde was related to greater rainfall rates and parental moisture from the southeastern Caribbean basin.

\section{Conclusions}

Stable isotope signatures in precipitation and water vapor are useful indicators of climate variability and provide critical information regarding regional and global water processes. In temperate regions, isotopic sampling has been conducted for decades, providing novel insights on water cycle dynamics. However, isotopic records from the humid tropics, such as Central America, have been quite limited to date. Long-term $\delta^{18} \mathrm{O}$ and $\delta^{2} \mathrm{H}$ records 
NOAA HYSPLIT MODEL

Backward trajectories ending at 2300 UTC 18 Jul 11 GDAS Meteorological Data

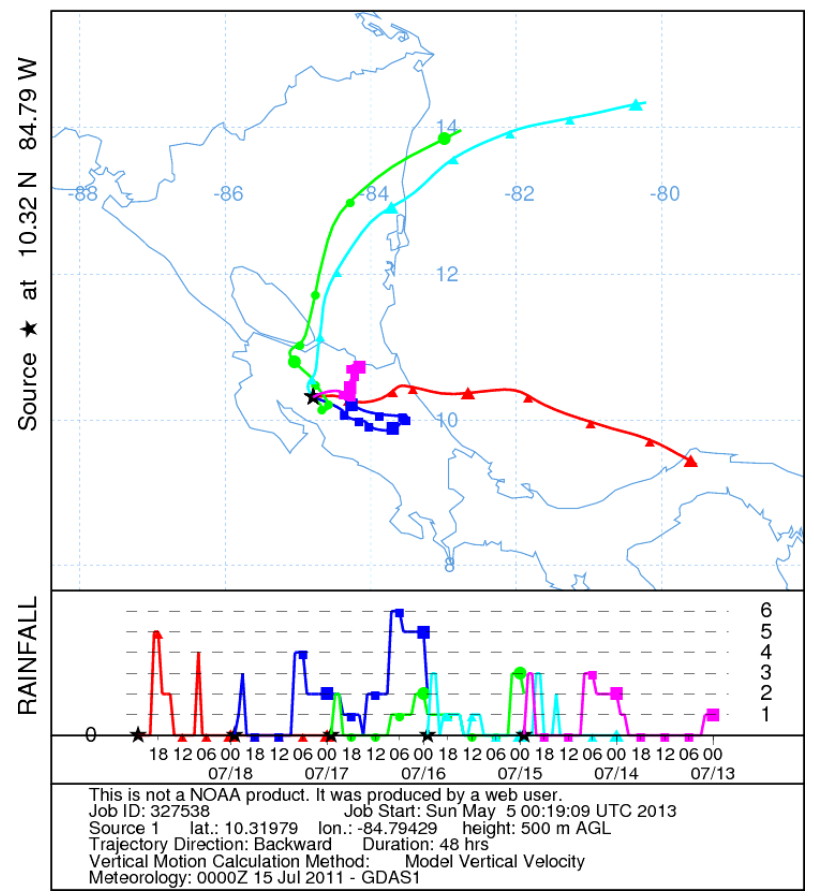

(a)

NOAA HYSPLIT MODEL

Backward trajectories ending at 2300 UTC 26 Jul 11 GDAS Meteorological Data

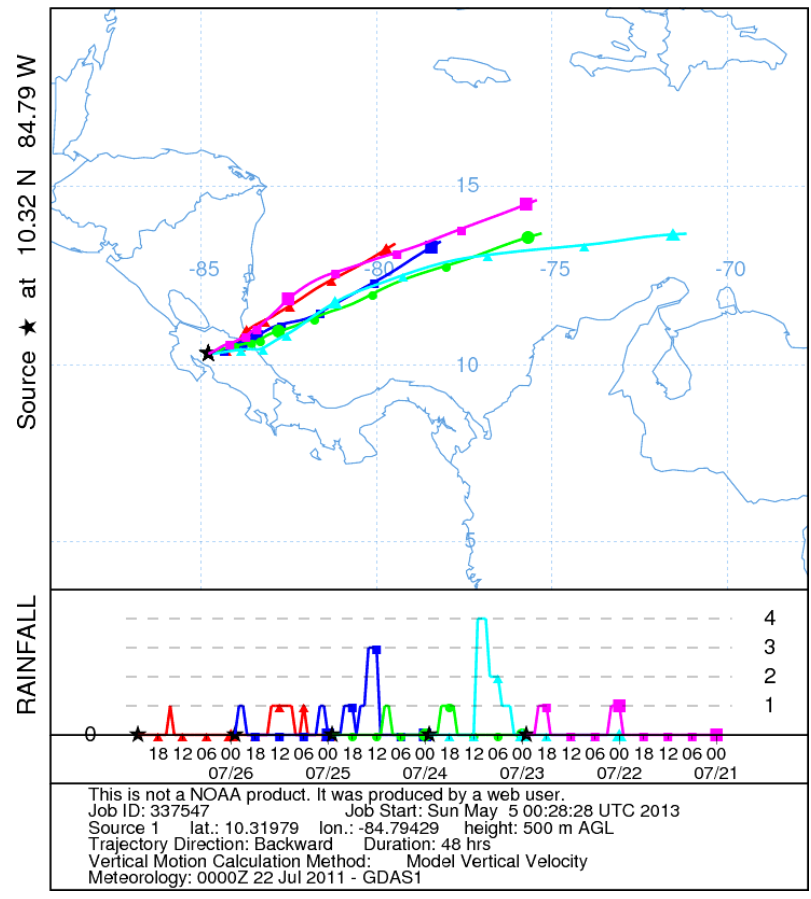

(c)
NOAA HYSPLIT MODEL

Backward trajectories ending at 2300 UTC 17 Oct 11

GDAS Meteorological Data

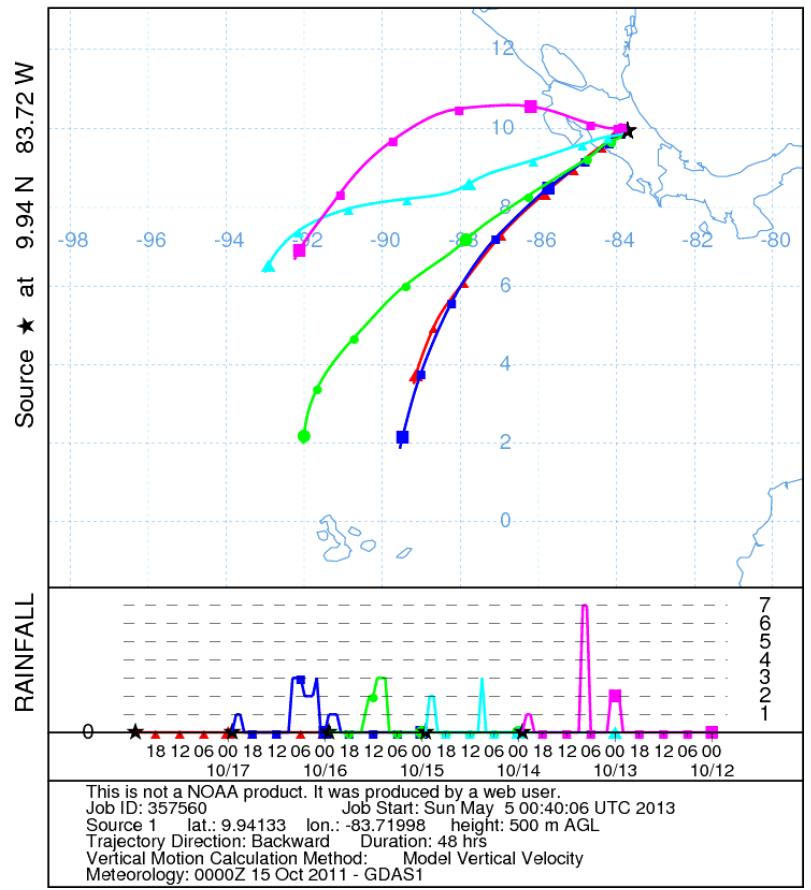

(b)

NOAA HYSPLIT MODEL

Backward trajectories ending at 2300 UTC 27 Oct 11

GDAS Meteorological Data

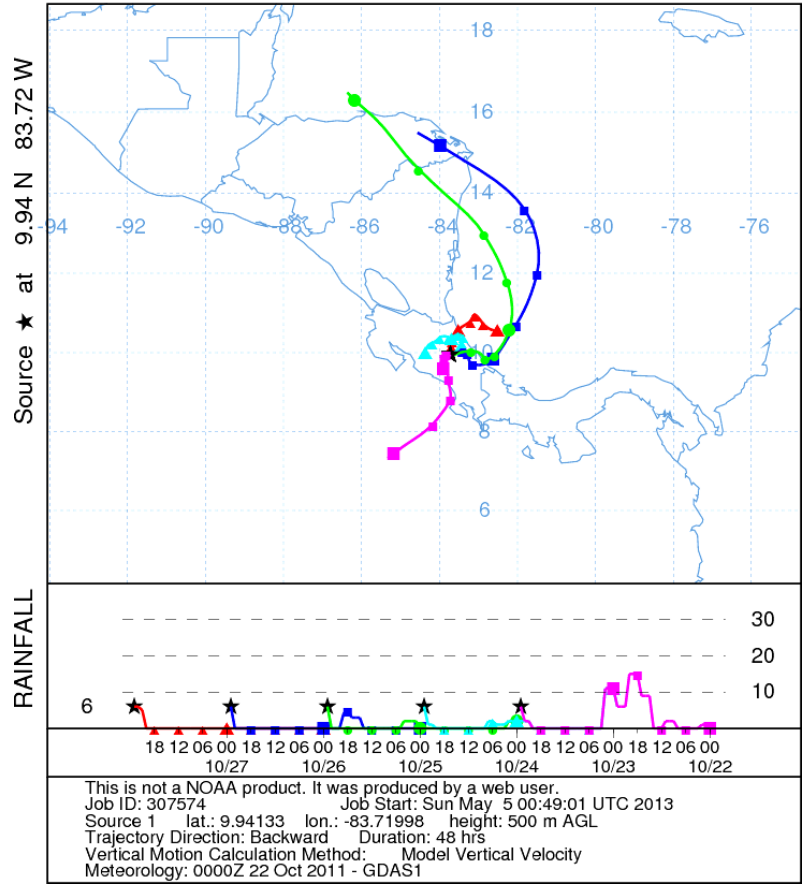

(d)

Figure 9. HYSPLIT model simulations including rainfall intensity in mm/hr. Depleted events: (a) Monteverde 7/18/11 and (b) Turrialba 10/17/11. Enriched events: (c) Monteverde 7/26/11 and (d) Turrialba 10/27/11. Five-day air mass back trajectories are color coded. 
offer a unique opportunity to study precipitation and ground and surface water processes at a relatively low cost compared to continuous streamflow or climate monitoring, which is often not feasible in tropical developing countries due to high costs and limited resources. Based on historic GNIP records, we conducted a spatial and temporal characterization of stable isotopes in four main geographical regions of Costa Rica: Nicoya Peninsula, Pacific Coast, Caribbean slope, and Central Valley. Despite data limitations several correlations were found. The regression analysis of precipitation amounts with annual arimethic means in samples from all four regions yields a slope of $-1.6 \%$ o $\delta^{18} \mathrm{O}$ per $100 \mathrm{~mm}$ of rain $\left(r^{2}=\right.$ $0.57)$, which corresponds with a temperature effect of $-0.37 \%$ o $\delta^{18} \mathrm{O} /{ }^{\circ} \mathrm{C}$. A strong correlation $\left(r^{2}=0.77\right)$ of $-2.0 \% \delta^{18} \mathrm{O}$ per $\mathrm{km}$ of elevation was also found in these data.

$D$-excess has been identified as a potential indicator of recycled moisture. Therefore, meteoric $d$-excess isoscapes coupled with isotopic monitoring in surface waters may provide quantitative estimates of the contribution of recycled moisture, which would greatly enhance our understanding of water budgets in Costa Rica. $D$-excess interpolation from 46 GNIP stations suggests that evaporation enrichment often occurs over the Nicoya Peninsula, especially during periods of lowest precipitation (January-April). The Nicoya Peninsula, which receives less than $1500 \mathrm{~mm}$ annually, has been recently identified [44] as a hydrologically vulnerable region due to the increase of water demand and susceptibility to experience prolonged drought periods. Greater $d$-excess values were observed in the inter-mountainous region of the Central Valley and the Pacific slope. It is still unknown if moisture recycling is occurring due to evaporation within dense canopy covers (e.g., Zurquí-Irazú or Monteverde areas) or if instead moisture is available from multiple sources such as flood plains, dams or reservoirs, or soil moisture. However, this moisture input may play a significant role in late afternoon and overnight convective storms along the Pacific slope.

The illustrative example using the HYSPLIT model indicates that enriched isotopic compositions both in Turrialba and Monteverde are related to central Caribbean parental moisture and small rainfall intensities. Depleted events appear to be related to high rainfall amounts despite the parental moisture origin. Further event-based sampling in strategic locations across the country coupled with air mass trajectory analysis is needed to evaluate the effects of parental moisture origin and isotope signatures. Overall, we believe that this preliminary stable isotope analysis across Costa Rica may provide a useful baseline for future isotopic studies in the region as well as enhance our understanding of moisture recycling processes within tropical inter-mountainous systems. Further research should evaluate spatial and temporal isotopic variations in surface and groundwater systems to ultimately determine critical recharge zones across the country.

\section{Acknowledgements}

We thank the International Atomic Energy Agency for granting access to historic GNIP records. A National Science Foundation-IGERT scholarship (Grant No. 0903479) US Borlaug Fellowship for Food Security supported the isotopic fieldwork in Turrialba, Costa Rica. Funding provided by the Chemistry School at the National University of Costa Rica and the National University Council of Costa Rica was essential to acquire a laser water isotope analyzer. The authors are also grateful for access to the field sites and logistical support by the Tropical Science Center and the Monteverde Cloud Forest Reserve.

\section{REFERENCES}

[1] P. Kabat, R. E. Schulze, M. E. Hellmuth and J. A. Veraart, "Coping with Impacts of Climate Variability and Climate Change in Water Management: A Scoping Paper," DWCReport No. DWCSSO-01 International Secretariat of the Dialogue on Water and Climate, Wageningen, 2003.

[2] R. K. Colwell, G. Brehm, C. L. Cardelús, A. C. Gilman and J. T. Longino, "Global Warming, Elevational Range Shifts, and Lowland Biotic Attrition in the Wet Tropics," Science, Vol. 322, No. 5899, 2008, pp. 258-261. http://dx.doi.org/10.1126/science.1162547

[3] S. R. Loarie, P. P. Duffy, H. Hamilton, G. P. Asner, C. B. Field and D. D. Ackerly, "The Velocity of Climate Change," Nature, Vol. 462, No. 7276, 2009, pp. 1052-1055. http://dx.doi.org/10.1038/nature08649

[4] J. J. Tewksbury, R. B. Huey and C. A. Deutsch, "Putting the Heat on Tropical Animals," Science, Vol. 320, No. 5881, 2008, pp. 1296-1297. http://dx.doi.org/10.1126/science.1159328

[5] Y. P. Zhou, K. M. Xu, Y. C. Sud and A. K. Betts, "Recent Trends of the Tropical Hydrological Cycle Inferred from Global Precipitation Climatology Project and International Satellite Cloud Climatology Project Data," Journal of Geophysical Research, Vol. 116, No. D6, 2011, Article ID: D09101. http://dx.doi.org/10.1029/2010JD015197

[6] V. Magaña, J. A. Amador and S. Medina, "The Midsummer Drought over Mexico and Central America," Journal of Climate, Vol. 12, No. 6, 1999, pp. 1577-1588. http://dx.doi.org/10.1175/1520-0442(1999)012<1577:TM DOMA $>2.0 . \mathrm{CO} ; 2$

[7] G. Bowen, "Statistical and Geostatistical Mapping of Precipitation Water Isotope Ratios," In: J. B. West, et al., Eds., Isoscapes: Understanding Movement, Pattern, and 
Process on Earth through Isotope Mapping, Springer Science, New York, 2010, pp. 139-160. http://dx.doi.org/10.1007/978-90-481-3354-3 7

[8] L. J. Araguás-Araguás, K. O. Froehlich and K. Rozanski, "Stable Isotope Composition of Precipitation over Southeast Asia," Journal of Geophysical Research, Vol. 103, No. D22, 1998, pp. 28721-28742. http://dx.doi.org/10.1029/98JD02582

[9] K. M. Cobb, J. F. Adkins, J. W. Partin and B. Clark, "Regional-Scale Climate Influences on Temporal Variation of Rainwater and Cave Dripwater Oxygen Isotopes in Northern Borneo," Earth and Planetary Science Letters, Vol. 263, No. 3-4, 2007, pp. 207-220. http://dx.doi.org/10.1016/j.epsl.2007.08.024

[10] Y. Ishizaki, K. Yoshimura, S. Kanae, M. Kimoto, N. Kurita and T. Oki, "Interannual Variability of $\mathrm{H}_{2}{ }^{18} \mathrm{O}$ in Precipitation over the Asian Monsoon Region," Journal of Geophysical Research, Vol. 117, No. D16, 2012, Article ID: D16308. http://dx.doi.org/10.1029/2011JD015890

[11] K. R. Johnson and B. L. Ingram, "Spatial and Temporal Variability in the Stable Isotope Systematics of Modern Precipitation in China: Implications for Paleoclimate Reconstructions," Earth and Planetary Science Letters, Vol. 220, No. 3-4, 2004, pp. 365-377. http://dx.doi.org/10.1016/S0012-821X(04)00036-6

[12] M. Lachniet, "Sea Surface Temperature Control on the Stable Isotopic Composition of Rainfall in Panama," Geophysical Research Letters, Vol. 36, No. 3, 2009, Article ID: L03701. http://dx.doi.org/10.1029/2008GL036625

[13] M. Lachniet and W. Patterson, "Oxygen Isotope Values of Precipitation and Surface Waters in Northern Central America (Belize and Guatemala) Are Dominated by Temperature and Amount Effects," Earth and Planetary Science Letters, Vol. 284, No. 3, 2009, pp. 435-446. http://dx.doi.org/10.1016/j.epsl.2009.05.010

[14] M. Vuille, R. S. Bradley and F. Keimig, "Interannual Climate Variability in the Central Andes and Its Relation to Tropical Pacific and Atlantic Forcing," Water Resources Research, Vol. 105, No. D10, 2000, pp. 12447-12460. http://dx.doi.org/10.1029/2000JD900134

[15] M. Vuille, R. S. Bradley and F. Keimig, "Climatic Variability in the Andes of Ecuador and Its Relation to Tropical Pacific and Atlantic Sea Surface Temperature Anomalies," Journal of Climate, Vol. 13, No. 14, 2000, pp. 2520-2535.

http://dx.doi.org/10.1175/1520-0442(2000)013<2520:CV ITAO $>2.0 . \mathrm{CO} ; 2$

[16] M. Vuille, R. S. Bradley, R. Healy, M. Werner, D. R. Hardy, L. G. Thompson and F. Keimig, "Modeling d18O in Precipitation over the Tropical Americas 2: Simulation of the Stable Isotope Signal in Andean Ice Cores," Journal of Geophysical Research, Vol. 108, No. D6, 2003, p. 4175. http://dx.doi.org/10.1029/2001JD002039

[17] K. Ichiyanagi and M. D. Yamanaka, "Interannual Variation of Stable Isotopes in Precipitation at Bangkok in Response to El Niño Southern Oscillation," Hydrological Processes, Vol. 19, No. 17, 2005, pp. 3413-3423.

\section{http://dx.doi.org/10.1002/hyp.5978}

[18] H. O. Panarello and C. Dapeña, "Large Scale Meteorological Phenomena, ENSO and ITCZ, Define the Paraná River Isotope Composition," Journal of Hydrology, Vol. 365, No. 1-2, 2009, pp. 105-112.

http://dx.doi.org/10.1016/j.jhydrol.2008.11.026

[19] M. Vuille and M. Werner, "Stable Isotopes in Precipitation Recording South American Summer Monsoon and ENSO Variability: Observations and Model Results," Climate Dynamics, Vol. 25, No. 4, 2005, pp. 401-413. http://dx.doi.org/10.1007/s00382-005-0049-9

[20] P. K. Aggarwal, O. A. Alduchov, K. O. Froehlich, L. J. Araguas-Araguas, N. C. Sturchio and N. Kurita, "Stable Isotopes in Global Precipitation: A Unified Interpretation Based on Atmospheric Moisture Residence Time," Geophysical Research Letters, Vol. 39, No. 11, 2012, Article ID: L11705. http://dx.doi.org/10.1029/2012GL051937

[21] L. J. Araguás-Araguás, K. O. Froehlich and K. Rozanski, "Deuterium and Oxygen-18 Isotope Composition of Precipitation and Atmospheric Moisture," Hydrological Processes, Vol. 14, No. 8, 2000, pp. 1341-1355. http://dx.doi.org/10.1002/1099-1085(20000615)14:8<134 1::AID-HYP983>3.0.CO;2-Z

[22] G. Bowen, "Spatial Analysis of the Intra-Annual Variation of Precipitation Isotope Ratios and Its Climatological Corollaries," Journal of Geophysical Research, Vol. 113, No. D5, 2008, Article ID: D05113. http://dx.doi.org/10.1029/2007JD009295

[23] W. Dansgaard, "Stable Isotopes in Precipitation," Tellus, Vol. 16, No. 4, 1964, pp. 436-468. http://dx.doi.org/10.1111/j.2153-3490.1964.tb00181.x

[24] K. Rozanski, L. J. Araguas-Araguas and R. Gonantini, "Isotopic Patterns in Modern Global Precipitation," In: P. K. Swart, K. C. Lohmann, J. McKenzie and S. Savin, Eds., Climate Change in Continental Isotopic Records, American Geophysical Union, Washington DC, 1993, pp. $1-36$.

[25] L. I. Wassenaar, P. Athanasopoulos and M. J. Hendry, "Isotope Hydrology of Precipitation, Surface and Ground Waters in the Okanagan Valley, British Columbia, Canada,' Hydrology, Vol. 411, No. 1, 2011, pp. 37-48. http://dx.doi.org/10.1016/j.jhydrol.2011.09.032

[26] G. Bowen and J. Revenaugh, "Interpolating the Isotopic Composition of Modern Meteoric Precipitation," Water Resources Research, Vol. 39, No. 10, 2003, p. 1299. http://dx.doi.org/10.1029/2003WR002086

[27] M. Lachniet and W. Patterson, "Stable Isotope Values of Costa Rican Surface Waters," Journal of Hydrology, Vol. 260, No. 1-4, 2002, pp. 135-150. http://dx.doi.org/10.1016/S0022-1694(01)00603-5

[28] G. J. Bowen, J. B. West and J. Hoogewerff, "Isoscapes: Isotope Mapping and Its Applications," Geochemical Exploration, Vol. 102, No. 3, 2009, pp. 5-7. http://dx.doi.org/10.1016/j.gexplo.2009.05.001

[29] V. Barras and I. Simmonds, "Observation and Modeling of Stable Water Isotopes as Diagnostics of Rainfall Dynamics over Southeastern Australia," Journal of Geophysical Research, Vol. 114, No. D23, 2009, pp. 1-17. 
http://dx.doi.org/10.1029/2009JD012132

[30] H. Celle-Jeanton, R. Gonfiantini, Y. Travi and B. Sol, "Oxygen-18 Variations of Rainwater during Precipitation: Application of a Rayleigh Model to Selected Rainfalls in Southern France," Journal of Hydrology, Vol. 289, No. 1-4, 2004, pp. 165-177. http://dx.doi.org/10.1016/j.jhydrol.2003.11.017

[31] T. B. Coplen, P. J. Neiman, A. B. White, J. M. Landwehr, F. M. Ralph and M. D. Dettinger, "Extreme Changes in Stable Hydrogen Isotopes and Precipitation Characteristics in a Landfalling Pacific Storm," Geophysical Research Letters, Vol. 35, No. 21, 2008, Article ID: L21808. http://dx.doi.org/10.1029/2008GL035481

[32] N. C. Munksgaard, C. M. Wurster, A. Bass and M. I. Bird, "Extreme Short-Term Stable Isotope Variability Revealed by Continuous Rainwater Analysis," Hydrological Processes, Vol. 26, No. 23, 2012, pp. 3630-3634. http://dx.doi.org/10.1002/hyp.9505

[33] J. Reynolds-Vargas and J. Fraile, "Use of Stable Isotopes in Precipitation to Determine Recharge Areas within the Barva Aquifer, Costa Rica," Latinoamerican Stable Isotopes Studies, International Atomic Energy Agency, Viena, 2006, pp. 83-96.

[34] P. Waylen, C. N. Caviedes and M. F. Quesada, "Interannual Variability of Monthly Precipitation in Costa Rica," Journal of Climate, Vol. 9, No. 10, 1996, pp. 2506-2613. http://dx.doi.org/10.1175/1520-0442(1996)009<2606:IV $\underline{\mathrm{OMPI}>2.0 . \mathrm{CO} ; 2}$

[35] A. J. Guswa, A. Rhodes and S. E. Newell, "Importance of Orographic Precipitation to the Water Resources of Monteverde, Costa Rica," Advances in Water Resources, Vol. 30, No. 10, 2007, pp. 2098-2112. http://dx.doi.org/10.1016/j.advwatres.2006.07.008

[36] GNIP, "Global Network of Isotopes in Precipitation," 2012.

http://wwwnaweb.iaea.org/napc/ih/IHS_resources_gnip.html
[37] Instituto Tecnológico de Costa Rica (ITEC), “Atlas de Costa Rica. Escuela de Ingeniería Forestal. Cartago, Costa Rica,” Digital Atlas, ITEC, Cartago, Costa Rica, 2008.

[38] R. R. Draxler and G. D. Rolph, "HYSPLIT-Hybrid Single Particle Lagrangian Integrated Trajectory Model," 2013. http://ready.arl.noaa.gov/HYSPLIT.php

[39] H. Craig, "Isotopic Variations in Meteoric Waters," Science, Vol. 133, No. 3465, 1961, pp. 1702-1703. http://dx.doi.org/10.1126/science.133.3465.1702

[40] C. Leibundgut, P. Maloszewski and C. Kulls, "Tracers in hydrology," Wiley-Blackwell, Oxford, 2009.

[41] R. Gonfiantini, M. A. Roche, J. C. Olivry, J. C. Fontes and G. M. Zuppi, "The Altitude Effect on the Isotopic Composition of Tropical Rains," Chemical Geology, Vol. 181, No. 1, 2001, pp. 147-167. http://dx.doi.org/10.1016/S0009-2541(01)00279-0

[42] M. A. Scholl, J. B. Shanley, J. P. Zegarra and T. B. Coplen, "The Stable Isotope Amount Effect: New Insights from NEXRAD Echo Tops, Luquillo Mountains, Puerto Rico," Water Resources Research, Vol. 45, No. 12, 2009 , Article ID: W12407. http://dx.doi.org/10.1029/2008WR007515

[43] A. L. Rhodes, A. J. Guswa and S. E. Newell, "Seasonal Variation in the Stable Isotopic Composition of Precipitation in the Tropical Montane Forests of Monteverde, Costa Rica," Water Resources Research, Vol. 42, 2006, Article ID: W11402. http://dx.doi.org/10.1029/2005WR004535

[44] MINAET-IMN, "Mejoramiento de las Capacidades Nacionales para la Evaluación de la Vulnerabilidad y Adaptación del Sistema híDrico al Cambio climÁtico en Costa Rica, Como Mecanismo para Disminuir el Riesgo al Cambio climÁtico y Aumentar el Índice de Desarrollo Humano," In: J. A. Retana, Eds., Ministerio de Ambiente, Energía y Telecomunicaciones, Instituto Meteorológico Nacional San José, Costa Rica, 2012, 46 p. 